\title{
Seeing Shapes and Hearing Textures: Two Neural Categories of Touch
}

\author{
Miriam Reiner ${ }^{\#, 1,2}$, Maria Stylianou-Korsnes ${ }^{*, \dagger, \#, 3,4}$, Gary Glover ${ }^{5}$, Kenneth Hugdahl ${ }^{6}$ and \\ Marcus W. Feldman ${ }^{2}$ \\ ${ }^{I}$ Technion, Israel Institute of Technology, Haifa, Israel \\ ${ }^{2}$ Department of Biological Sciences, Stanford University, Stanford, USA \\ ${ }^{3}$ Oslo University Hospital, Ullevål, Department of Psychiatry of Old Age, Asker, Norway \\ ${ }^{4}$ Department of Psychology, Stanford University, Stanford, USA \\ ${ }^{5}$ Department of Radiology, Stanford University School of Medicine, Stanford, USA \\ ${ }^{6}$ Department of Biological and Medical Psychology, University of Bergen and Division of Psychiatry, Haukeland \\ University Hospital, Bergen, Norway
}

\begin{abstract}
Touching for shape recognition has been shown to activate occipital areas in addition to somatosensory areas. In this study we asked if this combination of somatosensory and other sensory processing areas also exist in other kinds of touch recognition. In particular, does touch for texture roughness matching activate other sensory processing areas apart from somatosensory areas? We addressed this question with functional magnetic resonance imaging (fMRI) using wooden abstract stimulus objects whose shape or texture were to be identified. The participants judged if pairs of objects had the same shape or the same texture. We found that the activated brain areas for texture and shape matching have similar underlying structures, a combination of the primary motor area and somatosensory areas. Areas associated with object-shape processing were activated between stimuli during shape matching and not texture roughness matching, while auditory areas were activated during encoding of texture and not for shape stimuli. Matching of textures also involves left BA47, an area associated with retrieval of relational information. We suggest that texture roughness is recognized in a framework of ordering. Left-lateralized activations favoring texture might reflect semantic processing associated with grading roughness quantitatively, as opposed to the more qualitative distinctions between shapes.
\end{abstract}

Keywords: Haptic, auditory, visual, ordering, texture, shape.

\section{INTRODUCTION}

Imagine looking for a keyhole in the dark. You touch the wooden door until you feel the smooth metal texture of the key-lock. Then, using the fingers, you identify the keyhole shape and insert the key. The fingers are used for both texture and shape recognition. Touch activities for both seem similar, yet the information conveyed is different. Most studies on touch report on overlapping activations within the somatosensory areas for texture and shape processing (e.g. $[1])$, while shape specific $[2,3]$ and texture specific regions have been suggested [2, 4]. However, some of these differences may just as well depend on differences in touch involved (e.g., palm of hand for shape / fingers for textures). Even though similar stimuli are expected to lead to similar neural activations, most studies that have used similar stimuli for both shape and texture recognition, report on activation of occipitotemporal visual areas in touch-for-shape recognition but not in touch-for-texture recognition (e.g. [5]).

*Address correspondence to this author at the Oslo University Hospital, Ullevål, department of psychiatry of old age, Slottsberget 35, 1385 Asker Norway, Tel +47 90023549, Fax: 669088 98;

E-mail: m.s.korsnes@psykologi.uio.no

"These authors contributed equally to this work.

'Current address: Oslo University Hospital, Department of Psychiatry of Old Age, Slottsberget 35, Asker, Norway.
One study also reported a texture specific region in the medial occipital cortex for both visual and haptic stimuli [2].

The visual processing of objects in the brain can be divided into a ventral stream (what), which extends from the visual cortex to the inferior temporal cortex and the dorsal stream (how/where) which extends from the visual cortex to the parietal lobes [6]. Object recognition and location by touch activates a corresponding dorsal stream from early somatosensory to prefrontal, inferior and superior parietal regions, respectively [7]. Recognition of graspable objects involves occipitotemporal areas, in particular the lateral occipital tactile-visual area $([2,5,8,9]$. This area is activated for shape recognition but not for texture recognition $[2,5,8$, 10-12]. This indicates that these areas respond to object form regardless of sensory input $[5,13,14]$, however the relative involvement of these areas may be modulated by properties such as object familiarity $[15,16]$. In addition, the cerebellum is more activated in the memory delay of tactile object recognition than for texture recognition, perhaps due to reflection over object shape processing.

Similar multisensory integration is also evident between tactile and auditory stimuli; when studying tactile texture perception it is common to take measures to prevent feeling textures from being biased due to auditory stimulation (e.g. $[17,18]$, such measures includes use of earplugs, head- 
phones, and a proper baseline. Tactile vibrations to the participant's index finger have shown to activate auditory areas $[5,19,20]$, however, this area of research is much less explored. With respect to tactile exploration, it is possible that an audiotactile activation overlap within Brodmann area (BA 42) reflect the bumpy exploration of a texture, or perhaps the dynamic contact between the hand and the stimulus [5, 20]. Animal studies on monkeys also show that this type of tactile vibration overlap with auditory stimulation in the second stage of auditory processing [21, 22]. Thus, we hypothesize that just as shape recognition rely on areas normally associated with visual processing, texture recognition rely on areas normally associated with auditory processing.

Neural correlates of kinesthetic and tactile exploration are difficult to distinguish; both cause overlapping activation in somatosensory areas and in the primary motor area [3]. We expect that the kinesthetic used in following the boundaries of a shape with a finger involve the generation of a mental image that may be detected as specific activation in the visual brain areas, however such mental images are products of further processing of sensory inputs, thus it is interesting to determine the sequences of activations that correspond uniquely to each of texture and shape recognition. Vision probably has a role in generating visual images but may not be crucial - blind people are able to draw images of objects, and sighted people can draw images with their eyes closed. Shape and texture roughness are two different kinds of object properties of general perceptual significance: shape is qualitative while texture roughness can be associated with quantitatively grading roughness, and is therefore orderable. There are other properties of texture (stickiness, hardness, temperature) which must be kept at constant for all stimuli when studying texture roughness.

We expect that activations within somatosensory areas should be involved in both shape and texture matching, but that shape recognition but not texture recognition should involve visual shape-specific lateral occipital culcus (LOC) $[2,5,8,10,11]$, while we predict that areas within the Superior Temporal Gyrus (STG) may be involved in distinguishing between different textures [20].

\section{METHODS}

\section{Participants}

Sixteen right-handed college students (nine females), with an age range 21- 29 years (average 25 years), were recruited through sign-up sheets and received $\$ 40$ for their participation. The subjects were judged fit to participate in the fMRI experiment after completing a comprehensive medical questionnaire, and were tested for handedness with the Edinburgh Inventory [38]. They gave informed consent for a protocol approved by the Human Subjects Review Board at Stanford University. The tasks were practiced first outside and later inside the scanner. After the experimental sessions, the participants filled out a questionnaire concerning their strategies used to identify the tactile stimuli.

\section{Design and Materials}

We emphasize the similarity of sensory inputs during shape and texture exploration; both are similar tasks in which right-handed participants use their right pointing finger to follow the contour or texture roughness of an object in

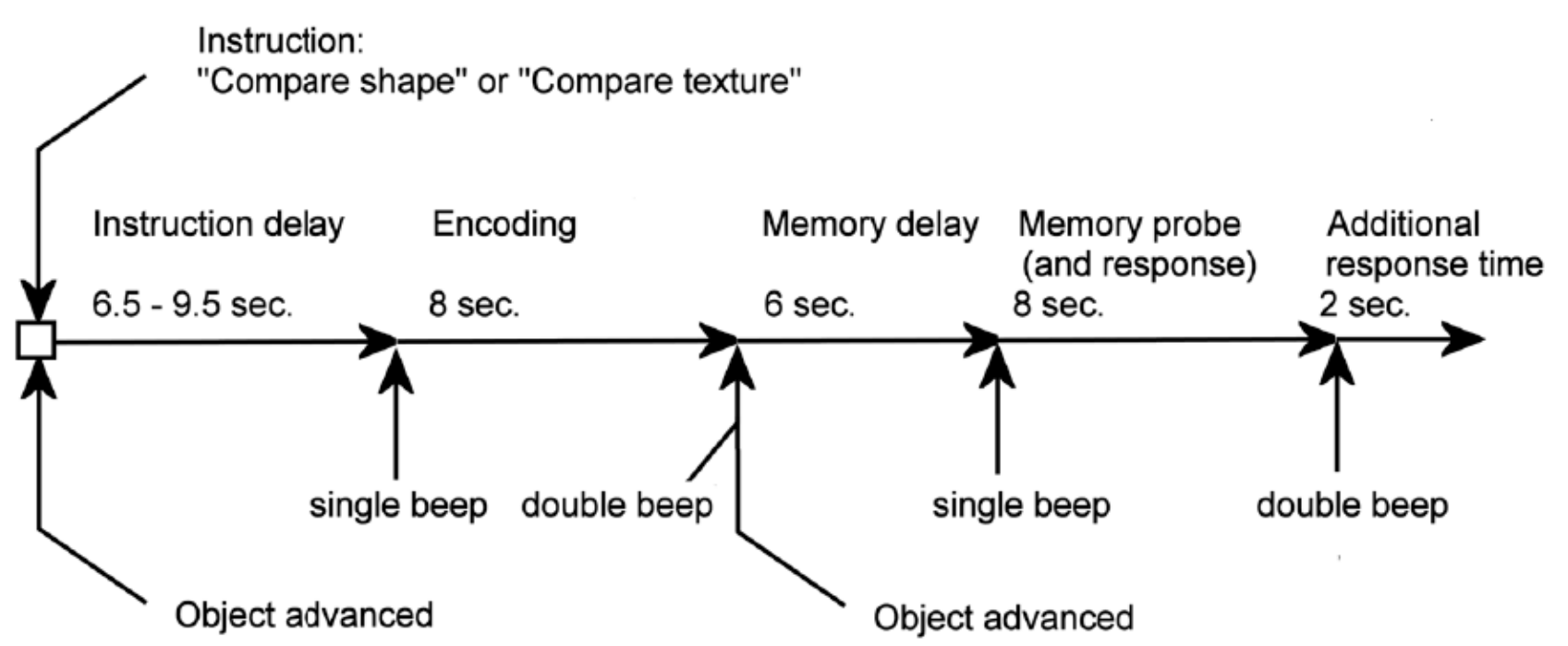

Fig. (1). Experimental design.

The participants were instructed to keep their eyes closed throughout the experiment, and auditory instructions ("compare texture" or "compare shape") set their minds to the following task. After an instruction-delay of variable duration (6.5, 7.5, 8.5 or 9.5 seconds; average 8seconds), an auditory signal prompted the participants to feel either the object's texture, or to feel the object's contour shape. A double auditory signal instructed them to stop. After a memory delay of 6 seconds during which the object was advanced to the next probe object, an auditory signal prompted the participants to feel the memory probe for 8 seconds, before a new double signal instructed them to stop. Participants responded "same" or "different" to whether the probe was identical to the encoded object by pressing one of two buttons with their left middle finger for one condition and the left pointing finger for the other condition, instructions were reversed for half the participants. The participants were instructed to respond as precisely as possible and as soon they were confident of their response (They were allowed to respond from the onset of the memory probe until 2 seconds after the memory probe. The item was then removed). The experiment consisted of six sessions; eight trials per scan, each trial lasting 32 seconds. 
order to decide whether the sensed feature of an object is identical to or different from that of the previous object. Hence our participants carry out similar tasks for recognition of shape and texture.

Stimulus objects, whose shape and texture were to be identified, were cut wooden abstract shapes, four shapes covered with four grades of sandpaper resulting in 16 unique shape/texture combinations. Each object was replicated in six exemplars, producing a total of 96 stimuli. The testobjects were attached to round carrousel turntables, $16 \mathrm{ob}-$ jects on each carrousel. The turntable was mounted on a nonmagnetic gearbox positioned between the participant's legs, and a wooden table was positioned over the turntable with a cutout through which the participant could touch only one object at a time with his/her pointing finger. The gearbox was connected through a shaft to a stepper motor to advance the objects under the cutout as commanded by the computer program developed for this purpose. Due to the limited capacity of the carrousels, the experiment was divided into six scans, with eight trials in each. Six carrousels containing the stimuli were prepared for each subject prior to scanning, and the carrousel was changed between each scan. The experiment was two-back-counterbalanced, so that each trial followed another with equal likelihood, with the constraint that each scan contained the same number of texture and shape trials. The order of stimuli was randomized between participants. The participants were instructed to keep their eyes closed throughout the experiment, and auditory instructions ("compare texture" or "compare shape") set their minds to the following task. After an instruction-delay of variable duration $(6.5,7.5,8.5$ or 9.5 seconds; average 8 -seconds), an auditory signal prompted the participants to feel either the object's texture, or to feel the object's contour shape. A double auditory signal instructed them to stop. After a memory delay of 6 seconds during which the object was advanced to the next probe object, an auditory signal prompted the participants to feel the memory probe for 8 seconds, before a new double signal instructed them to stop. Participants responded "same" or "different" to whether the probe was identical to the encoded object by pressing one of two buttons with their left middle finger for one condition and the left pointing finger for the other condition, instructions were reversed for half the participants. The participants were instructed to respond as precisely as possible and as soon they were confident of their response (They were allowed to respond from the onset of the memory probe until 2 seconds after the memory probe. The item was then removed). The experiment consisted of six sessions; eight trials per scan, each trial lasting 32 seconds for detailed procedure see Fig. (1). The detailed fMRI procedure is presented in the appendix.

\section{Questionnaire}

We included a questionnaire in order to evaluate whether or not the participants had understood the tasks, and in order to get a subjective measure on the strategies used in order to

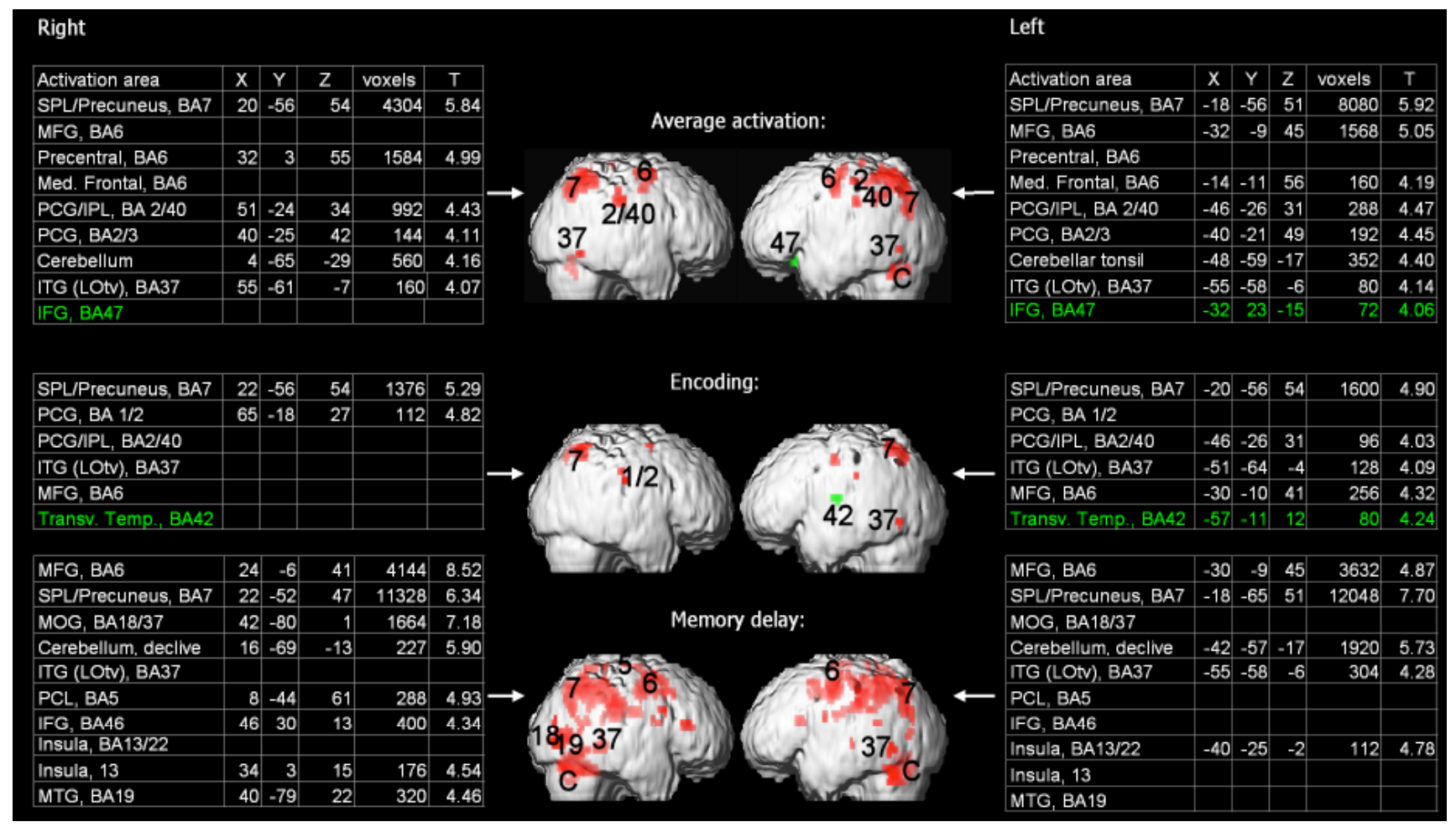

Fig. (2). Average brain activations of texture and shape recognition: for all phases and for each phase.

The figure show activation areas for shape - texture (red) and texture - shape (green) at the $\mathrm{P}=0.001$ threshold, uncorrected for multiple comparisons. Thus, by using the same stimuli and the same procedure for touch, auditory noise during scanning is controlled for when subtracting shape from texture activation and texture from shape activation.

Numbers on the figure are BA numbers; C: Cerebellum; SPL: Superior Parietal Lobe; MFG: Middle Frontal Gyrus: PCG: Post Central Gyrus; IPL: Inferior Parietal Lobule; ITG: Inferior Temporal Gyrus; LOtv: lateral occipital tactile-visual area: PcL: Paracentral Lobule; SFG: Superior Frontal Gyrus; STG: Superior Temporal Gyrus 
solve the tasks. For each task (shape or texture discrimination) the following questions were asked: 1) Did you experience any difficulty in discrimination between the stimuli? 2) How many different stimuli were there? 3) Did you have problems with any particular stimulus? 4) Did you use any particular strategies in order to discriminate one stimulus from the other? 5) Do you have any other comments to the stimuli? 6) Was the task easy or difficult? We also included a question about the perceived relative difficulty of the two tasks: 7) Which of the tasks was the easier task?

\section{RESULTS}

\section{Behavioral Results}

Participants recognized shapes with an average of $91 \%$ correct responses (SE: $2 \%$ ) and textures with an average of 85\% (SE: 2\%) correct responses. A t-test showed higher accuracy for "shape" decisions than for "texture" decisions $(\mathrm{t}(15)=2.788, \mathrm{p}<0.05)$. Since the participants were not asked to respond before they were confident of their answer, response time (RT) is not a reliable measure in this experiment.

\section{Evaluation of the Questionnaire}

None of the participants expressed difficulty in discriminating the textures, but 5 participants reported that the number of textures was lower than the actual number (they estimated 3.6 textures on average). Two participants did not report having used any strategies, while 11 categorized the stimuli by roughness and 3 named them. One participant expressed difficulty in discriminating the shapes, but he actually performed well. All participants could recall the shapes by drawing them, but 4 participants estimated the number of shapes to be higher than the actual number (they estimated it to be 4.4 shapes on average). Three participants reported not to have used any strategies, while all the others reported to have formed mental images and named or labeled the shapes. Half the participants (8) were more confident about their performance on the texture task than on the shape task, yet the accuracy results show they were less accurate on the texture task.

\section{fMRI Results}

The linear average activation over all four phases showed that the shape task activated several areas significantly more than the texture task. Only one area (left inferior frontal gyrus, BA 47) was activated more by texture than shape recognition, when all phases/conditions were included in the analysis (Fig. 2).

The shape task activated more regions than the texture task particularly during the memory delay, and especially in the occipitotemporal area. Both primary and secondary somatosensory (parietal operculum, BA40) regions were activated more during the shape task than the texture task. A ventral right lateral primary somatosensory region (PCG; BA $1,65-1827$ ) was activated significantly more during encoding of shapes than for textures. For the memory delay phase, shape specific local maxima were found in areas that have been reported as visual object shape- or size-related [23-25], including bilateral superior parietal lobule, (SPL; BA7, 5), bilateral precuneus (BA7) and middle frontal gyrus, (BA 6). Activations during the memory delay also included the left and the right inferior temporal gyrus (BA 37) within the area LOtv, which is associated with touch and/or visual shape recognition $[5,9,10,13,26]$. The shape recognition task activated more occipitotemporal regions than texture recognition (Fig. 2).

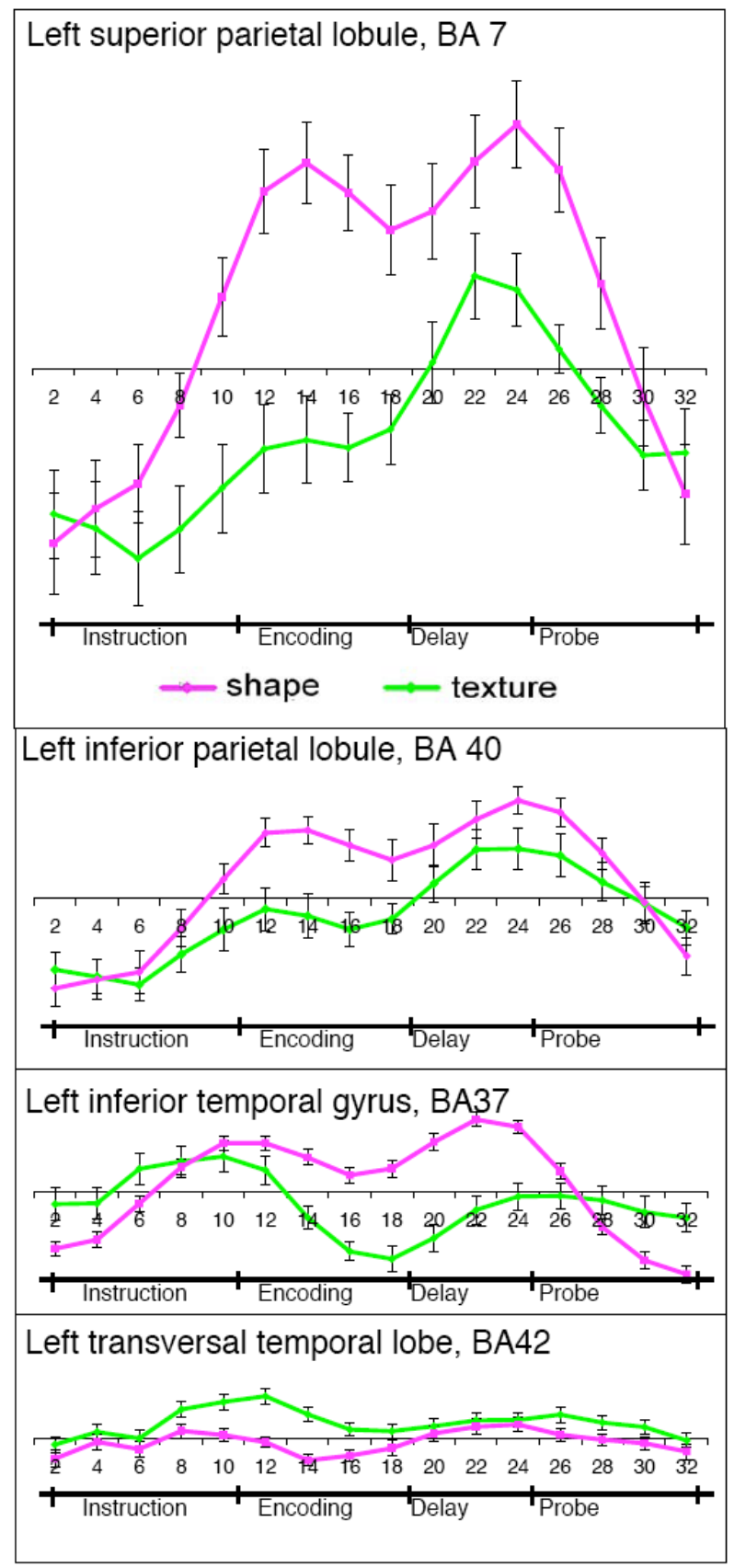

Fig. (3). Delayed HRF (haemodynamic response function) for areas of particular interest.

The y-axis shows percent signal change from average activation within the same ROI, all on the same scale $(\mathrm{x}$-axis $=0$, max peak $=$ $+/-1.5 \%)$. Bilateral regions within the superior parietal lobule (SPL) and the inferior parietal lobule (IPL) were consistently activated more for shape than for texture for all phases of the experiment. The inferior temporal region (LOC) was more activated for shape than for texture during the encoding phase and maintained during the memory delay. The area within the transverse temporal gyrus (BA42) was activated more for texture than for shape during the encoding phase. 
The left interior prefrontal cortex, LIPFC, (BA 47) was differentially more activated during texture recognition than during shape recognition. The LIPFC is preferentially involved in retrieval of relational information and error estimation in simple quantitative (arithmetic) tasks [27], in addition to semantic processes [28]. Greater activation for the texture task also occurred in the left associative auditory cortex (BA $42 ;-57,-11,12$ ) during the initial exploration of the stimuli. This region is close to the Heschl's gyrus and overlaps a region which activated for speech reading in hearing participants but not in deaf participants ([29]; -58, -3, 9), showing that this region is part of a hearing network. It is also in the proximity of a region showing overlap between tactile vibration to the index finger and auditory stimulation $(-44,-33$, 12 ), due to overlapping clusters with centers $-51,-21,16$ for vibrotactile stimulation and $-45,-21,4$ for audio [20].

Fig. (3) shows the difference between shape and texture activation within the left superior parietal lobule, the left inferior parietal lobule, the left inferior temporal gyrus and the left transverse temoral gyrus as a function of time.

There were several common activations in somatosensory areas (see Table 1), but no activation of anterior PCG (BA 3, $37-32$ 61), which has been reported to be a common region central in shape and texture identification [1]. However, the present experiment activated several common areas for shape and texture matching within the primary somatosensory area (BA 2, 3), primary motor area (BA4) and somatosensory association area (BA 5).

\section{DISCUSSION}

The results suggest parallels in texture and shape recognition through touch: texture recognition is correlated with primary and secondary somatosensory and secondary auditory areas in addition to frontal lobe areas; shape recognition is correlated with shape specific activations in somatosensory areas (SPL, IPL, PCG) and occipital areas; the following expands on this claim.

\section{Characteristics of Texture Matching}

Stilla and Sathian [2] reported activations in a medial occipital region when contrasting shape and texture stimuli. In the present experiment, no activations in the occipital areas were higher during texture roughness recognition than during shape recognition. Activation difference between these two studies may be due to method of exploration (one finger in our experiment, palm and all fingers in [2]), differences in stimuli (constant shape or constant texture for the variable not being studied in [2], versus varying shape and texture for all stimuli in the present experiment), difference in method (locator task in [2], versus the present matching task), or previous exposure to visual textures may have triggered e.g. an occipital region in [2]. In the present experiment, activations occurred in the associative auditory cortex during encoding of texture when contrasted with encoding of shape, even though it is possible that some information about the object's texture also was relayed subconsciously during exploration of shape. This area is located close to the tip of the left Heschl's gyrus (BA 42: $-57,-11,12$ ), and has seldom been reported in relation to other than auditory stimuli (but see [20]). Since this activation is present during the exploration of stimuli and not during the memory delay, it is likely that it is due to reflect bottom up processing (classifying stimulus as more or less rough) and indicate that multisensory integration occur within this area [19]. This also implies that damage to this region may affect other aspects than hearing. Overlapping activations for auditory and somatosensory stimuli have been previously reported in the associative auditory areas of monkeys [21,22], and for tactile stimulation $[19,20]$, but this is to our knowledge the first time it is shown during exploration of texture.

A region within LIPFC (BA47), an area that is preferentially involved in retrieval of relational information [30] was significantly more activated for the texture task than for the shape task. This fits subjects' reports that they ordered objects according to 'more or less rough' during texture recognition. We interpret the observed activation of BA 47 as a correlate of the relational encoding and maintenance of textures in memory: roughness of textures is memorized by creating a relation of order of roughness, as more or less rough.

\section{Characteristics of Shape Matching}

The higher activation in the postcentral gyrus (PCG) for shape than for texture during exploration (encoding) of the objects $(65,-18,27 \&-46,-26,31)$, suggests that additional

Table 1. Conjunction Between Shape and Texture Activations Including BAs 1, 2, 3, 4, 5 \& 7

\begin{tabular}{|c|c|c|c|c|c|c|c|c|c|c|c|}
\hline & \multicolumn{6}{|c|}{ Left } & \multicolumn{5}{|c|}{ Right } \\
\hline Sup. Parietal Lobule & $7 / 5$ & -24 & -52 & 43 & 1488 & 6.54 & 28 & -48 & 43 & 576 & 5.18 \\
\hline Precuneus & 7 & -22 & -64 & 36 & 144 & 6.19 & 22 & -64 & 36 & 112 & 4.33 \\
\hline Postcentral Gyrus & 2 & -48 & -29 & 35 & 96 & 5.03 & & & & & \\
\hline Post/precentral gyrus & $3 / 4$ & -22 & -29 & 49 & 560 & 5.23 & 24 & -31 & 49 & 272 & 4.15 \\
\hline Precentral gyrus & 4 & & & & & & 59 & -10 & 26 & 144 & 4.11 \\
\hline Cuneus & 7 & -24 & -80 & 30 & 160 & 3.9 & & & & & \\
\hline
\end{tabular}


recourses in the primary somatosensory region are required for identification of object shape and not object texture, but these activation differences do not continue after the exploration period (memory delay). Several parietal regions (SPL, BA5, 7); Precuenus (BA7), IPL (BA40), are particularly more activated for the shape task than for the texture task. These regions are known to be important for sensory integration and association (e.g. [7]). Superior and Inferior parts of the parietal cortex (BA's 7, 40) have also been associated with reasoning during spatial imagery [31], and regions within SPL has been found to activate due to short term memory processing during tactile length exploration of objects and spatial reasoning [31, 32]).

Our study shows that shape matching is supported by additional somatosensory regions and occipitotemporal regions which activate significantly less during texture matching, which is in line with previous reports that shape but not texture recognition involves object-specific occipital regions [5, $8,9]$. These regions include primary (BA2, 3) and secondary (BA40) somatosensory regions. Patient studies [33, 34] have shown that large damage to regions within primary somatosensory area has caused micro-geometrical processing deficits, while damage to secondary somatosensory areas (e.g. BA40, BA43) has caused macro-geometrical processing deficits. This suggests that texture processing depends on primary somatosensory regions that are activated for both texture and shape, while secondary somatosensory regions aid shape processing, and damage to secondary somatosensory regions may be more detrimental to shape than to texture processing.

We interpret the occipitotemporal activations for shape and not for texture (a bilateral area in the parietotemporal junction (BA 39) as relating to mental imagery (a strategy reported by the participants) which forms in the LOC, and is continued in temporal areas during the memory delay. Evidence for early involvement of LOC was recently reported in a high density EEG study [35]. The use of mental imagery may be enforced since the few shapes were familiar to the subjects in the present study [15]. This suggests that the occipitotemporal areas process not only visual sensory inputs, but also the position of the finger over time in order to produce a mental representation of shape. Thus there may be a modulation between a top-down effect of visual imagery and a bottom-up effect in perceiving objects [8]. Also, since activation of the LOC enhances verbal memory in the blind, there is processing beyond visual imagery in the LOC [36]. Similar results are reported by others who show that verbal [26] or whole-palm-touch stimuli $([2,13]$, or index finger and thumb touch [8] are associated with occipitotemporal activations.

\section{Qualitative Versus Quantitative Processing}

The overall results indicate two distinct cortical networks: shape matching correlates with activations in somatosensory and occipitotemporal areas while texture matching correlates with activations in somatosensory and secondary auditory cortex. The involvement of the occipital regions is evident during the memory delay, thus these regions are likely being used for maintenance of information about object shape. The involvement of the auditory area during the initial exploration of stimuli indicates that it is being used for encoding purposes. The two networks are associated with different theoretical concepts - shape is qualitative, texture is quantitative and orderable, and hence relational.

Recognition through the fingers may be viewed as correlated with two prototypical types of processing and corresponding activations; a) somatosensory- occipitotemporal related to qualitative processing, and b) somatosensoryauditory, related to relational processing. The left-lateralized activations favoring texture might reflect semantic processing associated with quantitatively grading roughness, as opposed to the more qualitative distinctions between shapes. In a previous study of neural correlates of shape/texture visual recognition, results suggest too that the LIFPC is activated for texture more than for shape [37] suggesting a pattern of processing beyond modalities. The activation of the LIFPC for texture may be due to its orderable nature as more or less rough. Processing of shape and texture may exemplify two categories of concepts associated with sensory cues: qualitative-non-orderable such as shapes, and orderable-quantitative such as texture.

\section{APPENDIX \\ fMRI Procedure \\ MRI Scanning Procedure}

MR imaging was performed using a $1.5 \mathrm{~T}$ whole-body MRI scanner (General Electric Medical Systems Signa, Rev. 5.5, Waukesha, WI). A top-hat elliptical quadrature birdcage head-coil was positioned around the participant's head to obtain the MRI signal.

Head movement was minimized using a bite-bar, formed with each participant's dental impression. Functional images were obtained $\left(\mathrm{TR}=2000 \mathrm{~ms}\right.$; $\mathrm{TE}=40 \mathrm{~ms}$; flip angle $87^{\circ}$; $\mathrm{FOV}=220 \mathrm{~cm} 2 ; 64 \times 64$ voxels $)$ in the same slice locations used for anatomic images. These functional images contained BOLD contrast intensity values and were acquired continuously during task performance. The volumes covered the whole brain (23 contiguous slices, each $6 \mathrm{~mm}$ thick; 1 interleaved) and were acquired using a T2*-weighted 2D gradient-echo spiral pulse sequence, which is relatively insensitive to motion artifacts due to pulsatility. A total of 768 functional volumes were acquired for each participant. Three discarded volumes (a total of $6 \mathrm{~s}$ ) were acquired at the beginning of each session to allow for T1 stabilization. Finally, a high resolution $\mathrm{T} 1$-weighted multisliced anatomical image was acquired in axial orientation, using a 3D spoiled GRASS (SPGR) pulse sequence $(\mathrm{TR}=40 \mathrm{~ms}$; minimum TE; flip angle $15^{\circ} ; 124$ contiguous slices of $1.5 \mathrm{~mm}$ thickness; $256 \mathrm{x}$ 192 matrix; FOV $220 \mathrm{~cm} 2$ ).

\section{Data Preprocessing}

Image preprocessing and statistical analysis were performed using SPM99 (Wellcome Department of Cognitive Neurology, http://www.fil.ion.ucl.ac.uk/spm). To account for variable sampling times for the different slices, voxel time series were interpolated using sinc interpolation and were resampled using the first (bottom) slice as a reference point.

All T2*-weighted volumes were realigned to the first one in the time series to correct for between-scan motion. The structural T1-weighted volumes were spatially normalized to a standard MNI template in Talairach space using a 12- 
parameter affine normalization and 12 nonlinear iterations with $7 \times 8 \times 7$ basis functions. The spatial transformations derived from normalizing the structural volume taken in the functional acquisition plane were applied to the realigned T2*-weighted volumes. During normalization, all volumes were re-sampled in $2 \times 2 \times 4 \mathrm{~mm}$ voxels using sinc interpolation in space. Finally, all $\mathrm{T} 2 *$-weighted volumes were smoothed with a 7-mm full width at half-maximum isotropic Gaussian kernel to compensate for residual between-subject variability after spatial normalization, and to permit application of Gaussian random field theory to provide for corrected statistical inference.

\section{fMRI Analyses}

Initial exploratory analyses were performed to assess the magnitude of pair-wise differences between the different conditions at each voxel. As part of these analyses, further preprocessing steps were performed within SPM99. First, in order to remove low frequency drifts in the BOLD signal, the data were high-pass filtered with an average upper cutoff period of 115 seconds. Condition effects at each voxel were estimated according to the general linear model as implemented in SPM. The first scan in each session was omitted from the analysis in order to eliminate transient noise.

The data was analyzed with the general linear model, blocked into the 4 different phases (see Fig. 1). We report the linear average activation during the 4 phases and in addition activation during the initial exploration of shapes and textures (encoding), and also activations during the memory delay; this phase is of particular interest in order to assess the preparatory processing for memory performance. We report the differential activation between shape and texture recognition, which ensure that possible interference sources such as any auditory stimulation will be equated out of the analysis. We also report a conjunction analysis was performed on predefined areas including the motor and somatosensory areas (BAs 1 - 7), in order to identify common somatosensory activations between the shape and texture memory tasks. This conjunction was done without a baseline; multiple regression analysis without a constant term was performed on the individual unbalanced shape and texture activations prior to conjunction in SPM. Post hoc exploration of regions showing differences between shape and texture was performed using a time series analysis method (roimod1, Stanford University) where the each value is the average image intensity over the region of interest. The units reported reflect the "somewhat abstract" MR intensity. Time course starts with the onset of the first condition, and represents the average across every running of that trial. Each graph is plotted averages of all 16 subjects for each region of interest.

\section{REFERENCES}

[1] Servos P, Lederman S, Wilson D, Gati J. fMRI-derived cortical maps for haptic shape, texture, and hardness. Cogn Brain Res 2001; 12: 307-13.

[2] Stilla R, Sathian K. Selective visuo-haptic processing of shape and texture. Hum Brain Mapp 2008; 29:1123-38.

[3] Deibert E, Kraut M, Kremen S, Hart J. Neural pathways in tactile object recognition. Neurology 1999; 52: 113-41.

[4] Roland PE, O'Sullivan B, Kawashima R. Shape and roughness activate different somatosensory areas in the human brain. Proc Natl Acad Sci USA 1998; 95: 3295-300.
[5] Beauchamp MS. See me, hear me, touch me: multisensory integration in lateral occipital-temporal cortex. Curr Opin Neurobiol 2005; 15: $145-53$.

[6] Ungerleider LG, Mishkin M. Two cortical visual systems. In Analysis of visual behavior Ingle DJ, Goodale MA, Mansfield RJ, Eds. Cambridge: MIT Press 1982; pp. 549-86.

[7] Reed CL, Klatzky RL, Halgren E. What vs. where in touch: an fMRI study. Neuroimage 2005; 25: 718-26.

[8] Amedi A, Malach R, Hendler T, Peled S, Zohary E. Visuo-haptic object-related activation in the ventral visual pathway. Nat Neurosci $2001 ; 4: 324-30$.

[9] Amedi A, Jacobson G, Hendler T, Malach R, Zohay E. Convergence of visual and tactile shape processing in the human lateral occipital complex. Cereb Cortex 2002; 12: 1202-12.

[10] Grill-Spector K, Malach R. The human visual cortex. Annu Rev Neurosci 2004; 27: 649-77.

[11] Malach R, Reppas JB, Benson RR, et al. Object-related activity revealed by functional magnetic resonance imaging in human occipital cortex. Proc Natl Acad Sci USA 1995; 92: 8135-9.

[12] Cavina-Pratesi C, Kentridge RW, Heywood CA, Milner AD. Separate processing of texture and form in the ventral stream: Evidene from fMRI and visual agnosia. Cerebral Cortex 2010; 20: 433-46.

[13] Pietrini P, Furey ML, Ricciardi E, et al. Beyond sensory images: Object-based representation in the human ventral pathway. Proc Natl Acad Sci USA 2004; 101: 5658-63.

[14] Macaluso E, Driver J. Spatial attention and crossmodal interactions between vision and touch. Neuropsychol 2001; 39: 1304-16.

[15] Lacey S, Flueckiger P, Stilla R, Lava M, Sathian K. Object familiarity modulates the relationship between visual object imagery and haptic shape perception. Neuroimage 2010; 49: 1977-90.

[16] Deshpande G, Hu X, Lacey S, Stilla R, Sathian K. Object familiarity modulates effective connectivity during haptic shape perception. Neuroimage 2010; 49:1991-2000.

[17] Jousmäki V, Hari R. Parchment-skin illusion: sound-biased touch. Curr Biol 1998; 8(6): R190.

[18] Guest S, Catmur C, Lloyd D, Spence C. Audiotactile interactions in roughness perception. Exp Brain Res 2002; 146:161-71.

[19] Bolognini N, Papagno C, Moroni D, Maravita A. Tactile Temporal Processing in the Auditory Cortex. J Cogn Neurosci 2009; 22(6): 1201-11.

[20] Schürmann M, Caetano G, Hlushchuk Y, Jousmäki V, Hari R Touch activates human auditory cortex. Neuroimage 2006; 30: 1325-31.

[21] Kayser C, Petkov CI, Augath M, Logothetis NK. Integration of touch and sound in auditory cortex. Neuron 2005; 48: 373-84.

[22] Schroeder CE, Lindsley RW, Specht C, Marcovici A, Smiley JF, Javit DC. Somatosensory input to auditory association cortex in the macaque monkey. J Neurophysiol 2001; 85: 1322-7.

[23] Kellenbach ML, Brett M, Patterson K. Large, colorful, or noisy? Attribute- and modality-specific activations during retrieval of perceptual attribute knowledge. Cogn Affect Behav Neurosci 2001; 1: 207-21.

[24] Oliver RT, Thomson-Schill SL. Dorsal stream activation during retrieval of object size and shape. Cogn Affect Behav Neurosci 2003; 3: 309-22.

[25] Hirsch J, Moreno DR, Kim KHS. Interconnected large-scale systems for three fundamental cognitive tasks revealed by functional MRI. J Cogn Neurosci 2001; 13: 389-405.

[26] Newman SD, Klatzky RL, Lederman SJ, Just MA. Imagining material versus geometric properties of objects: an fMRI study. Cogn Brain Res 2005; 23: 235-46.

[27] Badgaiyan RD, Schacter DL, Alpert NM. Retrieval of relational information: a role for the left inferior prefrontal cortex. Neuroimage 2002; 17: 393-400.

[28] Fiez JA. Phonology, semantics, and the role of the left inferior prefrontal cortex. Hum Brain Mapp 1997; 5: 79-83.

[29] MacSweeney M, Campbell R, Calvert GA, et al. Dispersed activation in the left temporal cortex for speech-reading in congenitally deaf people. Proc R Soc Lond 2001; 268: 451-7.

[30] Menon V, Makenzie K, Rivera SM, Reiss AL. Prefrontal cortex involvement in processing incorrect arithmetic equations: evidence rrom event-related fMRI. Hum Brain Mapp 2002; 16: 119-30.

[31] Knauff M, Mulack T, Kassubek J, Salih HR, Greenlee MW. Spatial imagery in deductive reasoning: a functional MRI study. Cog Brain Res 2002; 13(2): 203-12. 
[32] Hartmann S, Missimer JH, Stoeckel C, et al. Functional Connectivity in Tactile Object Discrimination: a principal component analysis of an event related fMRI-study. PLoS ONE 2008; 3(12): e3831.

[33] Binkofski F, Kunesh E, Classen J, Seitz RJ, Freud HJ. Tactile apraxia. Unimodal apractic disorder of tactile object exploration associated with parietal lobe lesions. Brain 2001; 124:132-44.

[34] Bohlhalter S, Fretz C, Weder B. Hierarchical versus parallel processing in tactile object recognition: a behavioural-neuroanatomical study of aperceptive tactile agnosia. Brain 2002; 125: 2537-48.

[35] Lucan JN, Foxe JJ, Gomez-Ramirez M, Sathian K, Molholm S. Tactile shape discrimination recruits human lateral occipital com- plex during early perceptual processing. Hum Brain Mapp 2010; Doc.Id: $10.1002 / \mathrm{hbm} .20983$.

[36] Sadato N, Okada T, Honda M, Yonekura Y. Critical period for cross-modal plasticity in blind humans: a functional MRI study. Neuroimage 2001; 16: 389-400.

[37] Stylianou-Korsnes M, Reiner M, Magnussen SJ, Feldman MW. Visual recognition of shapes and textures: an fMRi study. Brain Struct Funct 2010; Doc.Id: 10.1007/s00429-010-0241-5.

[38] Oldfield RC. The assessment and analysis of handedness: the Edinburgh inventory. Neuropsychologia 1971; 9: 97-113.

(c) Reiner et al.; Licensee Bentham Open.

This is an open access article licensed under the terms of the Creative Commons Attribution Non-Commercial License (http://creativecommons.org/licenses/by-nc/3.0/) which permits unrestricted, non-commercial use, distribution and reproduction in any medium, provided the work is properly cited. 